\title{
Avoiding arithmetic progressions in cyclic groups
}

\author{
Lorenz \& Stephanie Halbeisen
}

\begin{abstract}
Stephanie Gloor und Lorenz Halbeisen lernten sich 1995 kennen, als sie beide Assistenten an der ETH Zürich waren. Vertieft wurde ihre Beziehung, während sie an der Universität Zürich promovierte und er Postdoc-Aufenthalte in der Normandie und in Katalonien absolvierte. Nach einem gemeinsamen zweijährigen Aufenthalt in Berkeley sind sie 2001 als kleine Familie nach dem anderweitig bekannten Belfast gezogen, wo sie kürzlich ein Nachdiplomstudium in Informatik abgeschlossen hat und er Dozent an der Queen's University Belfast ist.
\end{abstract}

\section{Introduction}

Van der Waerden's theorem tells us that for any colouring of the positive integers with two colours, there are arbitrarily long non-constant arithmetic progressions in one colour, i.e., for every length $\ell$ there are positive integers $a$ and $d$ such that all the numbers $a, a+$ $d, a+2 d, \ldots, a+(\ell-1) d$ have the same colour. Such arithmetic progressions are called monochromatic. As a consequence, for any positive integer $r$ there exists a positive integer $n$ such that each colouring of the numbers $1,2, \ldots, n$ with two colours contains a monochromatic non-constant arithmetic progression of length $r$. In other words, we cannot avoid arithmetic progressions of length $r$ in both colours simultaneously.

Let us try to colour the numbers $1,2, \ldots, 9$ with two colours in such a way that neither colour contains an arithmetic progression of length 3. Let $\bigcirc$ and $\bullet$ denote the two colours respectively. Without loss of generality we may assume that 1 is coloured $\bullet$. We now

Ein Satz von van der Waerden besagt, dass bei jeder Färbung der natürlichen Zahlen mit zwei Farben zu beliebigem $\ell \geq 1$ positive natürliche Zahlen $a, d$ existieren, so dass $a, a+d, a+2 d, \ldots, a+(\ell-1) d$ gleich gefärbt sind. In der vorliegenden Arbeit untersuchen die Autoren Variationen dieses Resultats: Sie ersetzen $\mathbb{N}$ durch die zyklische Gruppe $\mathbb{Z}_{n}$ der ganzen Zahlen modulo $n$. Hier gibt es keine „Randeffekte“; anderseits kann eine arithmetische Folge dieselbe Zahl mehrfach belegen. Gefragt wird nach der maximalen Grösse einer Teilmenge $A \subset \mathbb{Z}_{n}$, die keine arithmetische Folge der gegebenen Länge $r$ trägt. Beispielsweise ist diese maximale Grösse für $r=n$ gleich $n(1-1 / p)$, wobei $p$ die kleinste Primzahl ist, die $n$ teilt. 
proceed by colouring successively the numbers $2,3, \ldots$ such that neither colour contains an arithmetic progression of length 3 . This leads to the following graph:

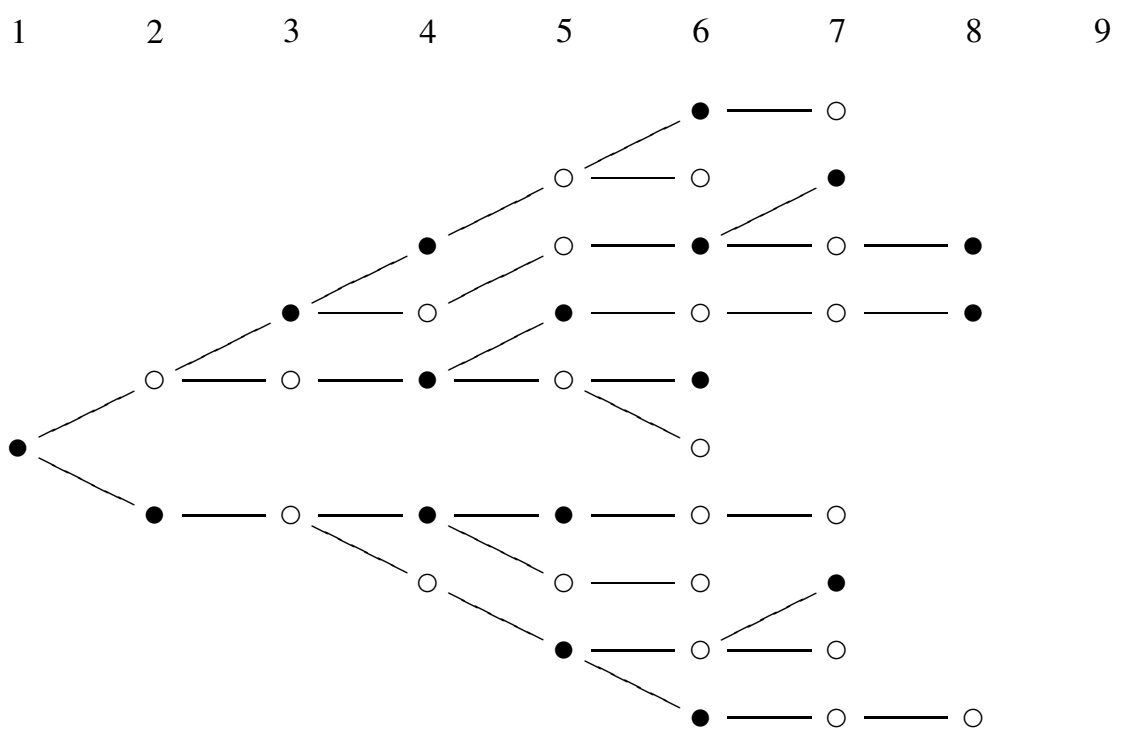

Firstly, this graph shows that it is possible to colour the numbers $1,2, \ldots, 8$ with two colours such that neither colour contains a non-constant arithmetic progression of length 3 . Secondly, we see that no matter how we colour the numbers $1,2, \ldots, 9$ with two colours, there is always a monochromatic non-constant arithmetic progression of length 3.

For given positive integers $n$ and $r$ we can always ask how large a subset of $\{1,2, \ldots, n\}$ may be such that it does not contain any arithmetic progression of length $r$. To find optimal upper bounds for the cardinality (i.e., size) of such a set is still an open problem, even in the case of $r=3$. In order to make the problem more symmetric and to avoid "boundary effects", we shall consider the cyclic set $(\mathbb{Z} / n \mathbb{Z})=\mathbb{Z}_{n}$, instead of the linear set $1,2, \ldots, n$, and ask for the maximum cardinality of a subset in $\mathbb{Z}_{n}$ which does not contain any non-constant arithmetic progression of a given length. More precisely, a nonconstant arithmetic progression with respect to $\mathbb{Z}_{n}$ of length $r$ is a non-constant sequence in the cyclic group $\left(\mathbb{Z}_{n},+\right)$ (i.e., modulo $n$ ) of the form $a, a+d, a+2 d, \ldots, a+(r-1) d$, where $a \in \mathbb{Z}_{n}$ and $1 \leq d<n$. Notice that we do not require all elements of the sequence to be different. Seeking for large sets in cyclic groups which do not contain arithmetic progressions of a given length leads to the following question:

Given a cyclic group $\mathbb{Z}_{n}$ and a positive integer $r$. What is the maximum cardinality of a set $A \subseteq \mathbb{Z}_{n}$ such that $A$ does not contain any non-constant arithmetic progression with respect to $\mathbb{Z}_{n}$ of length $r$ ?

In order to give partial answers to this question we shall use finite affine planes, a result in finite geometry, hypergraphs (a general form of graphs), a result for the linear case, as well as some combinatorics. 
First we like to reformulate our question above in terms of hypergraphs, but before we can do so, we have to introduce some terminology.

A hypergraph $H=(V, E)$ is a finite set $V$ of "vertices" together with a finite set $E$ of "edges" (sometimes called "hyperedges"), which are arbitrary non-empty subsets of $V$ (for a systematic study of hypergraphs we refer the reader to [1]). If all edges of a hypergraph $H$ have the same cardinality $r$, then the hypergraph $H$ is called $\boldsymbol{r}$-uniform. In particular, a graph without loops is a 2-uniform hypergraph. A hypergraph is called regular if all vertices belong to the same number of edges. A set of vertices of a hypergraph $H$ which does not (completely) contain any edge of $H$ is called an independent set. The complement of an independent set is a set of vertices which meets each edge of the hypergraph. Such a set is called a transversal. For a hypergraph $H$, the independence number $\alpha(H)$ (in the literature also called stability number) is the maximum cardinality of an independent set of $H$ (see [1]). The transversal number $\tau(H)$ of a hypergraph $H$ is the smallest cardinality of a transversal of $H$. If each vertex of $H$ is contained in at least one edge of $H$, then the complement of a maximal independent set (i.e., an independent set which is not properly contained in another independent set) is a minimal transversal (i.e., does not properly contain another transversal) and vice versa. In particular, we get that $\alpha(H)+\tau(H)$ is equal to the number of vertices of $H$.

Let us now turn back to our question:

For a positive integer $n$, we identify the elements of the cyclic group $\mathbb{Z}_{n}$ with the set $\{0,1, \ldots, n-1\}$. For a positive integer $r$ with $r \leq n$, let $H_{n, r}=\left(V_{n}, E_{r}\right)$ be the hypergraph defined as follows: $V_{n}:=\mathbb{Z}_{n}$ and a finite set $e \subseteq V_{n}$ belongs to $E_{r}$ if and only if there is a non-constant arithmetic progression $P$ in $\mathbb{Z}_{n}$ of length $r$, so that the elements appearing in $P$ are exactly the elements of $e$. Since $\mathbb{Z}_{n}$ is completely symmetric, $H_{n, r}$ is always a regular hypergraph, but, in general, $H_{n, r}$ is not $r$-uniform, e.g., $H_{4,3}=\{\{0,1,2\},\{1,2,3\},\{2,3,0\},\{3,0,1\},\{0,2\},\{1,3\}\}$. On the other hand, $H_{n, r}$ is always $r$-uniform for $n \geq r$ and $n$ prime. To see this, let $\left(a_{1}, a_{2}, \ldots, a_{r}\right)$ be an arithmetic progression with respect to $\mathbb{Z}_{n}$, where $n \geq r$ and $n$ prime. Let $d=a_{2}-a_{1}$ and assume that $a_{k}=a_{\ell}$ for some $1 \leq k<\ell \leq r$. Then $(k-\ell) d \equiv 0 \bmod n$, and since $n$ is prime, this implies that $d=0$ or $d=n$, and therefore, the sequence $\left(a_{1}, a_{2}, \ldots, a_{r}\right)$ is constant in $\mathbb{Z}_{n}$. Since the set of edges of $H_{n, r}$ corresponds to the set of all non-constant arithmetic progressions in $\mathbb{Z}_{n}$ of length $r$, it is easy to see that $\alpha\left(H_{n, r}\right)$ is equal to the maximum cardinality of a set $A \subseteq \mathbb{Z}_{n}$ such that $A$ does not contain any non-constant arithmetic progression of length $r$. So, to keep the notation short, let $\alpha(n, r):=\alpha\left(H_{n, r}\right)$.

For small numbers $n$ and $r$, the value $\alpha(n, r)$ can be easily calculated by computer, using for example a fast Prolog program. However, for general statements like $\alpha\left(p^{2}, p\right)=$ $(p-1)^{2}$ (for $p$ prime) we have to seek combinatorial proofs. The following result for hypergraphs gives us a lower bound on $\alpha(n, r)$ for $n>r$ and $n$ prime.

If $n>r$ and $H_{n, r}$ is $r$-uniform, e.g., if $n$ is prime, a lower bound for $\alpha(n, r)$ is given by the formula

$$
\alpha(n, r) \geq \frac{n}{m\left(H_{n, r}\right)^{1 / r}},
$$

where $m\left(H_{n, r}\right)$ denotes the number of edges of $H_{n, r}$ (see [1, p. 136]). Let us give some examples: For $n=7$ and $r=3$ we get $\alpha(7,3) \geq \frac{7}{21^{1 / 3}} \approx 2.54$, therefore, $\alpha(7,3) \geq 3$, 
and indeed, $\alpha(7,3)=3$. However, for $n=25$ and $r=5$ we get $\alpha(25,5) \geq \frac{25}{255^{1 / 5}} \approx 8.25$ and therefore, $\alpha(25,5) \geq 9$, but we will see later that $\alpha(25,5)=16$.

In the next section we will give some other lower bounds for $\alpha(n, r)$ and in the last section we will compute exact values of $\alpha(n, r)$ for certain numbers $n$ and $r$. As a matter of fact, we like to mention that $\alpha(n, r)$ is increasing in $r$, i.e., if $r \geq r^{\prime} \geq 1$, then $\alpha(n, r) \geq \alpha\left(n, r^{\prime}\right)$. But on the other hand, $\alpha(n, r)$ is not increasing in $n$. For example, $\alpha(19,3)=6$ but $\alpha(20,3)=5$.

\section{Lower Bounds}

For positive integers $n, a, r$ let $(n, a, r)$ be the following statement: There is a set $A \subseteq \mathbb{Z}_{n}$ of cardinality $a$ which does not contain any non-constant arithmetic progression of length $r$. As mentioned above, $\alpha(n, r)$ denotes the largest integer $a$ with $(n, a, r)$.

A set $A \subseteq \mathbb{Z}_{n}$ of cardinality $a$ witnesses $(n, a, r)$ if it does not contain any non-constant arithmetic progression of length $r$.

Remark If $B \subseteq \mathbb{Z}_{n}$ witnesses $(n, b, r)$ and $\alpha(n, r) \geq a>b$, then, in general, it is not true that there exists a set $A \supseteq B$ which witnesses $(n, a, r)$; or in terms of hypergraphs, not every maximal independent set of $H_{n, r}$ must have cardinality $\alpha\left(H_{n, r}\right)$. For example, $B=\{0,1,3,4,11,20\}$ witnesses $(27,6,3), \alpha(27,3)=8$, but there is no $A \supseteq B$ which witnesses $(27,7,3)$. A witness for $(27,8,3)$ is, for example, the set $\{0,1,3,4,9,10,12,13\}$.

Theorem 1.1. For all positive integers $n, m, a, b$ and $r$ we have:

$$
(n, a, r) \text { and }(m, b, r) \text { implies }(n m, a b, r) \text {. }
$$

Proof. For a sequence $\bar{z}=\left(z_{0}, \ldots, z_{n-1}\right)$ of 0's and 1's, let $\chi_{\bar{z}}:=\left\{i: z_{i}=1\right\} \subseteq \mathbb{Z}_{n}$. Further, let $\mathbf{0}_{n}=\underbrace{(0, \ldots, 0)}_{n-\text { times }}$. Suppose that $\bar{x}=\left(x_{0}, \ldots, x_{n-1}\right)$ and $\bar{y}=\left(y_{0}, \ldots, y_{m-1}\right)$ are such that $\chi_{\bar{x}}$ and $\chi_{\bar{y}}$ witness $(n, a, r)$ and $(m, b, r)$, respectively, then $\chi_{\bar{B}}$, where

$$
\bar{B}=\left(B_{y_{0}}, \ldots, B_{y_{m-1}}\right) \text { with } B_{y_{i}}= \begin{cases}\bar{x} & \text { if } y_{i}=1, \\ \mathbf{0}_{n} & \text { otherwise }\end{cases}
$$

witnesses $(n m, a b, r)$. Indeed, if $\chi_{\bar{B}}$ contains an arithmetic progression $\left(a_{1}, \ldots, a_{r}\right)$ of length $r$, then, since $\chi_{\bar{x}}$ witnesses $(n, a, r)$, the sequence $\left(a_{1} \bmod n, \ldots, a_{r} \bmod n\right)$ is constant. Thus, for every $1 \leq i \leq r$ we have $a_{i}=k_{i} \cdot n+c$, where $0 \leq k_{i}<m$ and $0 \leq c<n$. By construction, the $k_{i}$ 's belong to $\chi_{\bar{y}}$ and since $\chi_{\bar{y}}$ witnesses $(m, b, r)$, all the $k_{i}$ 's must be equal, and therefore, the sequence $\left(a_{1}, \ldots, a_{r}\right)$ is constant. Hence, $\chi_{\bar{B}}$ witnesses $(n m, a b, r)$, which completes the proof.

As an immediate consequence of Theorem 1.1 we get the following:

Corollary 1.2. For all positive integers $n, m$, and $r$ we have

$$
\alpha(n m, r) \geq \alpha(n, r) \cdot \alpha(m, r) .
$$


Remark In general, the lower bound for $\alpha(n m, r)$ given in Corollary 1.2 is not sharp. For example, $\alpha(4,4)=2$, but $\alpha(16,4)=6$, witnessed by $\{0,1,2,4,5,7\}$; and $\alpha(6,3)=2$, but $\alpha(36,3)=8$, witnessed by $\{0,1,3,4,9,10,12,13\}$. Moreover, this lower bound is not even sharp if $n$ and $m$ are two distinct prime numbers. For example, $\alpha(5,3)=2$ and $\alpha(7,3)=3$, but $\alpha(35,3)=9$, witnessed by $\{0,1,3,4,10,12,22,26,28\}$.

For any positive integers $n$ and $r \geq 3$, another lower bound for $\alpha(n, r)$ is given by the following:

Proposition 1.3. For any positive integers $n$ and $r$, where $r \geq 3$, we have

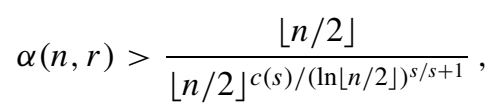

where $\lfloor n / 2\rfloor$ is the greatest integer which is less than or equal to $n / 2$, $s$ is a positive integer such that $2^{s}<r \leq 2^{s+1}$ and $c(s)>0$ is a constant depending only on $s$.

Proof. Let $m=\lfloor n / 2\rfloor,[m]=\{0,1, \ldots, m-1\}$ and let $v_{r}(m)$ be the cardinality of a largest set $A \subseteq[\mathrm{m}]$, so that $A$ does not contain any non-constant arithmetic progression with respect to $[m]$ of length $r$. Now, Robert Rankin proved in [5] that $v_{r}(m)>$ $m^{1-c(s) /(\ln m)^{s / s+1}}$, where $s$ is such that $2^{s}<r \leq 2^{s+1}$ and $c(s)$ is a constant depending only on $s$. Hence, if $A \subseteq[m]$ is such that $A$ does not contain any non-constant arithmetic progression with respect to $[m]$ of length $r$, then, since $n \geq 2 m$, A does not contain any non-constant arithmetic progression with respect to $\mathbb{Z}_{n}$ of length $r$, which completes the proof.

\section{Exact Values}

The table on the following page shows some exact values of $\alpha(n, r)$ for some small numbers $n$ and for $r=3$ and $r=5$, respectively. The values of $\alpha(n, r)$ as well as the witnesses we found with the help of the programming language Prolog.

In the following we compute the exact value of $\alpha(n, r)$ for certain positive integers $n$ and $r$. Let us begin with the case $n=r$.

Fact 2.1. If $p$ is prime, then $\alpha(p, p)=p-1$.

Proof. Obviously, we have $\alpha(p, p)<p$. On the other hand, since $p$ is prime, the set $\{0,1, \ldots, p-2\}$ witnesses $(p, p-1, p)$.

Theorem 2.2. If $n=m \cdot p$, where $p$ is the smallest prime number dividing $n$, then $\alpha(n, n)=m(p-1)=n(1-1 / p)$.

Proof. For each $h$ with $0 \leq h<m$, let $e_{h}:=\{h+m i: 0 \leq i<p\}$. Notice that each $e_{h}$ is equal to the set $h+m \mathbb{Z}_{p}=\left\{h+m i: i \in \mathbb{Z}_{p}\right\}$, which gives us an arithmetic preserving bijection between $\mathbb{Z}_{p}$ and $e_{h}$, and thus, each $e_{h}$ is an arithmetic progressions preserving copy of $\mathbb{Z}_{p}$. Therefore, each $e_{h}$ is an infinite non-constant arithmetic progression in $\mathbb{Z}_{n}$ with common difference $d=m$. Consider the hypergraph $H_{n, n}=\left(\mathbb{Z}_{n}, E_{n}\right)$, where $E_{n}$ is 


\begin{tabular}{|r|r||r|l|}
\hline \multicolumn{1}{|r||}{$n$} & $r$ & $\alpha(n, r)$ & \\
\hline 9 & 3 & 4 & $\{0,1,3,4\}$ \\
10 & 3 & 4 & $\{0,1,3,4\}$ \\
11 & 3 & 4 & $\{0,1,3,4\}$ \\
12 & 3 & 4 & $\{0,1,3,4\}$ \\
17 & 3 & 5 & $\{0,1,3,7,8\}$ \\
18 & 3 & 5 & $\{0,1,3,7,8\}$ \\
19 & 3 & 6 & $\{0,1,3,12,14,15\}$ \\
20 & 3 & 5 & $\{0,1,3,4,9\}$ \\
24 & 3 & 6 & $\{0,1,3,4,9,10\}$ \\
25 & 3 & 7 & $\{0,1,3,4,9,10,12\}$ \\
27 & 3 & 8 & $\{0,1,3,4,9,10,12,13\}$ \\
\hline 9 & 5 & 5 & $\{0,1,2,3,5\}$ \\
10 & 5 & 5 & $\{0,1,2,4,8\}$ \\
11 & 5 & 6 & $\{0,1,2,3,5,6\}$ \\
12 & 5 & 6 & $\{0,1,2,3,5,10\}$ \\
17 & 5 & 9 & $\{0,1,2,3,5,6,7,8,10\}$ \\
18 & 5 & 8 & $\{0,1,2,3,5,6,7,8\}$ \\
19 & 5 & 10 & $\{0,1,2,3,5,6,7,8,10,12\}$ \\
20 & 5 & 10 & $\{0,1,2,4,5,7,8,9,13,16\}$ \\
24 & 5 & 11 & $\{0,1,2,3,5,6,7,8,10,11,21\}$ \\
25 & 5 & 16 & $\{0,1,2,3,5,6,7,8,10,11,12,13,15,16,17,18\}$ \\
27 & 5 & 15 & $\{0,1,2,3,5,6,7,8,10,11,12,13,15,17,25\}$ \\
\hline & & & \\
\hline & 5 & & \\
1 & 5 & &
\end{tabular}

the set of all arithmetic progressions of length $n$ in $\mathbb{Z}_{n}$. Since each $e_{h}$ has $p$ elements and $p \leq n, e_{h} \in E_{n}$. Further, since $p$ is prime, by Fact 2.1 we have $\alpha(p, p)=p-1$, which implies that for any $j$ with $0 \leq j \leq p-1, e_{h} \backslash\{h+m j\} \notin E_{n}$. Finally, since there are $m$ edges $e_{h}$ and the $e_{h}$ 's are pairwise disjoint, we get $\alpha(n, n) \leq m(p-1)$.

On the other hand, the set $A=\{0,1, \ldots, m(p-1)-1\}$ witnesses $(n, m(p-1), n)$. Indeed, assume that $\left(a_{1}, \ldots, a_{n}\right)$ is a non-constant arithmetic progression with common difference $d<n$, built with elements of $A$. Since $|A|=m(p-1)$, the arithmetic progression uses at least one element of $A$ twice. Let $1<k_{0} \leq n$ be the least number such that $a_{1}=a_{k_{0}}=a_{1}+\left(k_{0}-1\right) d$. Then $\left(k_{0}-1\right) d=\ell n$, which implies that $\left\{a_{1}+k \frac{d}{\ell}: 0 \leq k<\right.$ $\left.k_{0}\right\}=\left\{a_{i}: 1 \leq i \leq k_{0}\right\}$. Because of the gap of length $m$ in $A, \frac{d}{\ell}>m$, but since $m$ is the greatest proper divisor of $n$, this is a contradiction.

Therefore we have $\alpha(n, n)=m(p-1)$, which completes the proof. 
As an immediate consequence of Theorem 2.2 we get the following:

Corollary 2.3. For any positive integer $m$ we have $\alpha(2 m, 2 m)=m$.

Moreover, combining Fact 2.1 and Corollary 1.2 we get the following:

Corollary 2.4. For any prime number $p$ and any non-negative integer $k, \alpha\left(p^{k}, p\right) \geq$ $(p-1)^{k}$.

Moreover, by Proposition 1.3, for large numbers $k$ and for prime numbers $p>2$ we have $\alpha\left(p^{k}, p\right)>(p-1)^{k}$. To see this, let $\varepsilon(k)=\frac{c(s)}{\left(\ln \left\lfloor\frac{p^{k}}{2}\right\rfloor\right)^{s / s+1}}$ and note that since $\lim _{k \rightarrow \infty} \ln \left\lfloor\frac{p^{k}}{2}\right\rfloor^{s / s+1} \rightarrow \infty$ we get $\lim _{k \rightarrow \infty} \varepsilon(k) \rightarrow 0$. Therefore, by taking the logarithm on both sides of the following expression, one verifies that for $k$ large enough we have

$$
\left(\left\lfloor\frac{p^{k}}{2}\right\rfloor\right)^{1-\varepsilon(k)}>(p-1)^{k},
$$

and since $\alpha\left(p^{k}, p\right)>\left(\left\lfloor\frac{p^{k}}{2}\right\rfloor\right)^{1-\varepsilon(k)}$ (by Proposition 1.3), it follows that for $k$ large enough we have $\alpha\left(p^{k}, p\right)>(p-1)^{k}$. Thus, the lower bound for $\alpha\left(p^{k}, p\right)$ given in Corollary 2.4 is, in general, not sharp. On the other hand, this lower bound is sharp for $k=2$. Before we can prove this result we have to introduce some terminology.

An affine plane of order $\boldsymbol{p}$, where $p$ is prime, is a set $P$ containing $p^{2}$ points, together with $p+1$ so-called parallel classes consisting of subsets of $P$ which are called lines, such that the following hold:

(i) Each parallel class contains $p$ pairwise disjoint lines.

(ii) Each line contains $p$ points of $P$.

(iii) For any two distinct points of $P$, there is exactly one line in some parallel class which contains these two points.

Theorem 2.5. For any prime number $p$ we have $\alpha\left(p^{2}, p\right)=(p-1)^{2}$.

Proof. By Corollary 2.4 we get $\alpha\left(p^{2}, p\right) \geq(p-1)^{2}$. Now, Robert Jamison in [3] and Andries Brouwer and Alexander Schrijver in [2] have shown that a set which intersects each line of the affine plane of order $p$, i.e., a transversal, must contain at least $2 p-1$ points. Notice, that the complement of a set which intersects each line of an affine plane cannot contain a line. Thus, in order to prove Theorem 2.5 it is enough to prove that there exists an affine plane of order $p$ such that each line forms an arithmetic progression with respect to $\mathbb{Z}_{p^{2}}$, since we then can conclude that $\alpha\left(p^{2}, p\right) \leq p^{2}-(2 p-1)=(p-1)^{2}$.

So, let us show that there is an affine plane of order $p$ such that every line forms an arithmetic progression of length $p$. Let $\left\{a_{i, j}: 0 \leq i, j<p\right\}$ be the set of points, where $a_{i, j}:=i+j p$ (for all $0 \leq i, j<p$ ). The $p^{2}+p$ lines $\ell$ are defined as follows: For $0 \leq j<p$ and $0 \leq s<p$ let $\ell_{j, s}:=\left\{a_{i,(s i+j) \bmod p}: 0 \leq i<p\right\}$ and let $\ell_{j, p}:=\left\{a_{j, i}:\right.$ $0 \leq i<p\}$. By construction, for fixed $j,\left\{\ell_{j, s}: 0 \leq s \leq p\right\}$ is the set containing the $p+1$ lines going through $a_{0, j}$, and for any $s$ with $0 \leq s \leq p$, the set $C_{s}=\left\{\ell_{j, s}: 0 \leq j<p\right\}$ 
consists of $p$ parallel lines, i.e., is a parallel class. Now, for every $0 \leq j<p$ and every $0 \leq s \leq p, \ell_{j, s}$ forms an arithmetic progression with respect to $\mathbb{Z}_{p^{2}}$. Indeed, for $0 \leq s<$ $p, \ell_{j, s}$ forms an arithmetic progression with common difference $s p+1$ and $\ell_{j, p}$ forms an arithmetic progression with common difference $p$. Further, for any two distinct points, there is exactly one line (in some parallel class $C_{s}$ ) which contains these two points.

Thus, for every prime number $p$ there exists an affine plane of order $p$ such that each line forms an arithmetic progression with respect to $\mathbb{Z}_{p^{2}}$, which completes the proof.

The proof of the Jamison-Brouwer-Schrijver result is algebraic, using polynomial equations over a finite field, and no combinatorial proof is known $\left(c f\right.$. [4, Problem $\left.\left.3.13^{+}\right]\right)$. In the case of $p=3$ or $p=5$, we were able to prove the equation $\alpha\left(p^{2}, p\right)=(p-1)^{2}$ in a purely combinatorial way. However, since the proof is already awkward for $p=5$, it is difficult to see how it could be extended to larger primes. In the following we like to present a combinatorial proof just for the case of $p=3$ :

Proposition 2.6. $\alpha(9,3)=4$.

Proof. By Corollary 2.4 we get $\alpha(9,3) \geq 4$. So, assume towards a contradiction that there is a set $\tilde{A} \subseteq \mathbb{Z}_{9}$ which witnesses $(9,5,3)$, or in other words, assume that $\tilde{A} \subseteq \mathbb{Z} 9$ is a set with five elements which does not contain any non-constant arithmetic progression of length 3. Let $M_{9}$ be the $3 \times 3$-matrix

$$
M_{9}=\left(\begin{array}{lll}
0 & 3 & 6 \\
1 & 4 & 7 \\
2 & 5 & 8
\end{array}\right)
$$

and let $R_{1}, R_{2}$ and $R_{3}$ be the rows of $M_{9}$. Since each row $R_{i}$ is equal to the set $i+3 \mathbb{Z}_{3}$, each row is an arithmetic progression preserving copy of $\mathbb{Z}_{3}$, and since $\alpha(3,3)=2$ for each $1 \leq i \leq 3$, we have $\left|\tilde{A} \cap R_{i}\right| \leq 2$ (where $\left|\tilde{A} \cap R_{i}\right|$ denotes the cardinality of the set $\left.\tilde{A} \cap R_{i}\right)$. Further, since $|\tilde{A}|=5$, there must be two rows, say $R_{1}$ and $R_{2}$, such that $\left|\tilde{A} \cap R_{1}\right|=\left|\tilde{A} \cap R_{2}\right|=2$, which - by checking the 9 possible cases - implies that $\tilde{A} \cap R_{3}=\emptyset$, and hence, $\tilde{A}$ does not witness $(9,5,3)$, which completes the proof.

As we have seen above, for any prime number $p>2$ and sufficiently large $k$ we have $\alpha\left(p^{k}, p\right)>(p-1)^{k}$. On the other hand, we also have seen that $\alpha\left(p^{2}, p\right)=(p-1)^{2}$ holds for any prime number $p$. Thus, it still might be the case that the equation $\alpha\left(p^{3}, p\right)=$ $(p-1)^{3}$ holds for all prime numbers $p$. A first step towards this conjecture is the following:

Proposition 2.7. $\alpha(27,3)=8$.

Proof. By Corollary 2.4 we get $\alpha(27,3) \geq 8$. So, assume towards a contradiction that there is a set $\tilde{A} \subseteq \mathbb{Z}_{27}$ which witnesses $(27,9,3)$. Let $M_{27}$ be the $3 \times 9$-matrix

$$
M_{27}=\left(\begin{array}{ccccccccc}
0 & 3 & 6 & 9 & 12 & 15 & 18 & 21 & 24 \\
1 & 4 & 7 & 10 & 13 & 16 & 19 & 22 & 25 \\
2 & 5 & 8 & 11 & 14 & 17 & 20 & 23 & 26
\end{array}\right)
$$

and let $R_{1}, R_{2}$ and $R_{3}$ be the rows of $M_{27}$. Since each row $R_{i}$ is an arithmetic progressions preserving copy of $\mathbb{Z}_{9}$ and $\alpha(9,3)=4$, for each $1 \leq i \leq 3,\left|\tilde{A} \cap R_{i}\right| \leq 4$. We partition 
$\mathbb{Z}_{27}$ into the three pairwise disjoint sets

$$
\begin{aligned}
& T_{1}=\{0,1,2,9,10,11,18,19,20\} \\
& T_{2}=\{3,4,5,12,13,14,21,22,23\} \\
& T_{3}=\{6,7,8,15,16,17,24,25,26\} .
\end{aligned}
$$

Let $j, k, l \in\{1,2,3\}$ be three distinct numbers. The three sets $T_{1}, T_{2}$ and $T_{3}$ are such that for each $i$ with $1 \leq i \leq 3$ we have

$$
R_{i} \cap \tilde{A} \cap T_{j} \neq \emptyset \text { and } R_{i} \cap \tilde{A} \cap T_{k} \neq \emptyset \text { implies } R_{i} \cap \tilde{A} \cap T_{l}=\emptyset .
$$

Indeed, let $a \in R_{i} \cap T_{j}$ and $b \in R_{i} \cap T_{k}$. Then, there are three different arithmetic progressions of length 3 through $a$ and $b$, say $\left\{a, b, c_{1}\right\},\left\{a, b, c_{2}\right\}$ and $\left\{a, b, c_{3}\right\}$, and by construction we have $\left\{c_{1}, c_{2}, c_{3}\right\}=R_{i} \cap T_{l}$. Since $|\tilde{A}|=9$, there must be two rows, say $R_{1}$ and $R_{2}$, such that $\left|\tilde{A} \cap R_{1}\right| \geq 3$ and $\left|\tilde{A} \cap R_{2}\right| \geq 3$. Hence, by ( $\left.\sharp\right)$, there must be $j_{1}, j_{2} \in\{1,2,3\}$ such that $\left|R_{1} \cap \tilde{A} \cap T_{j_{1}}\right|=\left|R_{2} \cap \tilde{A} \cap T_{j_{2}}\right|=2$. Consider the hypergraph $H_{27,3}=\left(\mathbb{Z}_{27}, E\right)$, where $E$ consists of all instances of arithmetic progressions of length 3 in $\mathbb{Z}_{27}$. For $a \in R_{1} \cap \tilde{A} \cap T_{j_{1}}$ and $b \in R_{2} \cap \tilde{A} \cap T_{j_{2}}$ let $S_{a, b}=\left\{c \in R_{3}:\{a, b, c\} \in E\right\}$. Then, $\left|S_{a, b}\right|=3$ and it is easy to see that $\left|S_{a, b} \cap T_{1}\right|=\left|S_{a, b} \cap T_{2}\right|=\left|S_{a, b} \cap T_{3}\right|=1$. Moreover, for $\left\{a_{1}, a_{2}\right\}=R_{1} \cap \tilde{A} \cap T_{j_{1}}$ and $\left\{b_{1}, b_{2}\right\}=R_{2} \cap \tilde{A} \cap T_{j_{2}}$ we have

$$
S_{a_{1}, b_{1}} \cup S_{a_{1}, b_{2}} \cup S_{a_{2}, b_{1}} \cup S_{a_{2}, b_{2}}=R_{3},
$$

which implies that $\tilde{A} \cap R_{3}=\emptyset$, and hence, $\tilde{A}$ does not witness $(27,9,3)$. Let us illustrate (*) with the following example: Let $j_{1}=1, a_{1}=0, a_{2}=9$, and $j_{2}=3$, and consider the six arithmetic progressions of length 3 going through $a_{1}$ or $a_{2}, R_{2} \cap T_{3}$, and $R_{3} \cap T_{3}$ :

\begin{tabular}{ccc|ccc}
$a_{1}$ & $R_{2} \cap T_{3}$ & $R_{3} \cap T_{3}$ & $a_{2}$ & $R_{2} \cap T_{3}$ & $R_{3} \cap T_{3}$ \\
\hline 0 & 7 & 17 & 9 & 7 & 8 \\
0 & 16 & 8 & 9 & 16 & 26 \\
0 & 25 & 26 & 9 & 25 & 17
\end{tabular}

Hence, no matter which two numbers $b_{1}$ and $b_{2}$ we take from $R_{2} \cap T_{3}$, we always have

$$
\left(R_{3} \cap T_{3}\right)=\left(S_{0, b_{1}} \cap T_{3}\right) \cup\left(S_{0, b_{2}} \cap T_{3}\right) \cup\left(S_{9, b_{1}} \cap T_{3}\right) \cup\left(S_{9, b_{2}} \cap T_{3}\right),
$$

which, by symmetry, is true for any choice of $a_{1}$ and $a_{2}$ from $R_{1} \cap T_{1}$. Thus, we have

$$
\left(R_{3} \cap T_{3}\right)=\left(S_{a_{1}, b_{1}} \cap T_{3}\right) \cup\left(S_{a_{1}, b_{2}} \cap T_{3}\right) \cup\left(S_{a_{2}, b_{1}} \cap T_{3}\right) \cup\left(S_{a_{2}, b_{2}} \cap T_{3}\right) .
$$

Considering the six arithmetic progressions of length 3 going through $a_{1}$ or $a_{2}, R_{2} \cap T_{2}$, and $R_{3} \cap T_{2}$, we get

$$
\left(R_{3} \cap T_{2}\right)=\left(S_{a_{1}, b_{1}} \cap T_{2}\right) \cup\left(S_{a_{1}, b_{2}} \cap T_{2}\right) \cup\left(S_{a_{2}, b_{1}} \cap T_{2}\right) \cup\left(S_{a_{2}, b_{2}} \cap T_{2}\right) .
$$

Similarly, by considering the six arithmetic progressions of length 3 going through $a_{1}$ or $a_{2}, R_{2} \cap T_{1}$, and $R_{3} \cap T_{1}$, we get

$$
\left(R_{3} \cap T_{1}\right)=\left(S_{a_{1}, b_{1}} \cap T_{1}\right) \cup\left(S_{a_{1}, b_{2}} \cap T_{1}\right) \cup\left(S_{a_{2}, b_{1}} \cap T_{1}\right) \cup\left(S_{a_{2}, b_{2}} \cap T_{1}\right) .
$$

Thus, we finally have

$$
R_{3}=\left(R_{3} \cap T_{1}\right) \cup\left(R_{3} \cap T_{2}\right) \cup\left(R_{3} \cap T_{3}\right)=S_{a_{1}, b_{1}} \cup S_{a_{1}, b_{2}} \cup S_{a_{2}, b_{1}} \cup S_{a_{2}, b_{2}} .
$$




\section{Summary}

The function $\alpha(n, r)$ is monotone in $r$ but not monotone in $n$. However, for any positive integers $n, m$ and $r$ we have $\alpha(n m, r) \geq \alpha(n, r) \cdot \alpha(m, r)$. In particular, for any positive integers $n, k$, and $r$ we have $\alpha\left(n^{k}, r\right) \geq \alpha(n, r)^{k}$, which implies that for any prime number $p, \alpha\left(p^{k}, p\right) \geq \alpha(p, p)^{k}=(p-1)^{\bar{k}}$. On the one hand, for each prime number $p>2$ there are integers $k$ such that $\alpha\left(p^{k}, p\right)>(p-1)^{k}$, but on the other hand, for every prime number $p$ we have $\alpha\left(p^{2}, p\right)=(p-1)^{2}$ and $\alpha(p, p)=(p-1)$. In addition, we have seen that $\alpha\left(3^{3}, 3\right)=2^{3}$ (Proposition 2.7) but the authors were not able to prove $\alpha\left(5^{3}, 5\right)=4^{3}$, since the proof of Proposition 2.7 seems not generalisable. This leads to the open question whether $\alpha\left(p^{3}, p\right)=(p-1)^{3}$ for all primes $p$ larger than 3 (the authors could not agree what they expect to be the answer). Further, we have seen that for any positive integer $n$, $\alpha(n, n)=n\left(1-\frac{1}{p}\right)$, where $p$ is the smallest prime number dividing $n$. In particular, for any positive integer $m$ we have $\alpha(2 m, 2 m)=m$.

\section{References}

[1] Berge, C.: Hypergraphs: Combinatorics of Finite Sets. North-Holland, Mathematical Library, vol. 45, Amsterdam 1989.

[2] Brouwer, A.E.; Schrijver, A.: The blocking number of an affine space. J. Combin. Theory Ser. A 24 (1978), 251-253.

[3] Jamison, R.E.: Covering finite fields with cosets of subspaces. J. Combin. Theory Ser. A 22 (1977), 253266.

[4] Jukna, S.: Extremal Combinatorics: With Applications in Computer Science. Springer-Verlag, Berlin et al. 2001.

[5] Rankin, R.A.: Sets of integers containing not more than a given number of terms in arithmetical progression. Proc. Roy. Soc. Edinburgh Sect. A 65 (1960/61), 332-344.

Lorenz Halbeisen

Department of Pure Mathematics

Queen's University Belfast

Belfast, BT7 1NN, Northern Ireland

e-mail: halbeis@qub.ac.uk

s.halbeisen@att.net 